\title{
An Eight-Way Radial Switch Based on SIW Power Divider
}

\author{
Dong-Mook Lee $\cdot$ Yong-Jun An $\cdot$ Jong-Gwan Yook
}

\begin{abstract}
This paper presents a single-pole eight-throw switch, based on an eight-way power divider, using substrate integrate waveguide(SIW) technology. Eight sectorial-lines are formed by inserting radial slot-lines on the top plate of SIW power divider. Each sectorial-line can be controlled independently with high level of isolation. The switching is accomplished by altering the capacitance of the varactor on the line, which causes different input impedances to be seen at a central probe to each sectorial line. The proposed structure works as a switching circuit and an eight-way power divider depending on the bias condition. The change in resonant frequency and input impedance are estimated by adapting a tapered transmission line model. The detailed design, fabrication, and measurement are discussed.
\end{abstract}

Key words: Power Divider, Radial Switch, SP8T, Tapered-Line Resonator.

\section{I . Introduction}

Switches play a major role in communication, radar, and commercial RF systems, such as a composition of feeding networks for a beam steering antenna system as well as signal routing. The duty of the switch is to open a target channel and isolate the remaining channels. This operation can be achieved either mechanically or electrically.

The mechanical waveguide switch, which uses short and open circuit loads in the waveguide, has good highpower handling capability, insertion loss, and isolation [1]. However, the switching speed is relatively slow compared to electrical switches, the switch is bulky in size, and it requires a complicated transition. On the other hand, electrical switches that use PIN diode or field effect transistor (FET) are easily integrated with a planar circuit. It only takes a few nano-seconds to control the switch on and off, depending on the characteristics of the components [2] [4]. The size issue may be alleviated by means of a planar switch circuit with solid state devices.

Planar single-pole multi-throw switches using PIN diodes have been reported for satellite communication systems [5] [7]. These planar structures mainly suffer from insufficient isolation. The switch circuit proposed in [5] requires the addition of shorted stubs to overcome low isolation while additional LC compensation has been utilized in [6].

Radio frequency microelectromechanical systems (RFMEMS) technology may provide improved performance compared to solid state devices and may be implementable in both planar [8] and waveguide structures [9]. However, RF MEMS switches have several drawbacks, such as high driven voltage, high cost of packaging, and reliability issues [10].

The general shape of a planar switch circuit, which is similar to the structures proposed in [5], [6], has a central coaxial feed, a circular disk for power distribution, and radially placed output transmission lines. The main advantage of these radially distributed lines is their ease of connection to beam forming array antennas and other systems. Nevertheless, re-routing the signals requires additional transmission lines to combine with other components such as a power combiner, amplifiers, and so on. The other sub networks, including quarter wave transformers, are also needed for deactivated lines to appear as an open circuit.

This paper presents a single-pole eight-throw switch network based on the SIW power divider that shows low insertion loss as well as an advantageous high level of integration [11] [13]. The proposed switch network consists of coaxial probes, which simplifies combination with other components compared to using a transmission line. This structure also does not require the additional short or open circuits presented in [5], [6], and can be

Manuscript received March 14, 2012 ; Revised July 4, 2012 ; Accepted August 14, 2012. (ID No. 20120314-008J)

Dept. of Electrical \& Electronic Engineering, Yonsei University, Seoul, Korea.

Corresponding Author : Jong-Gwan Yook (e-mail : jgyook@yonsei.ac.kr)

This is an Open-Access article distributed under the terms of the Creative Commons Attribution Non-Commercial License (http://creativecommons.org/licenses/ by-nc/3.0) which permits unrestricted non-commercial use, distribution, and reproduction in any medium, provided the original work is properly cited. 
easily fabricated by a standard PCB process. The analysis, simulation, and measurement of this switch are discussed in this paper.

\section{Design of the Radial Switch}

\section{2-1 Slot-added SIW Power Divider}

An eight-way radial cavity power divider, based on SIW technology, is first designed by following the procedure proposed in [11]. This power divider has one central coaxial probe and eight peripheral probes, along with the port numbering shown in Fig. 1(a). The annular
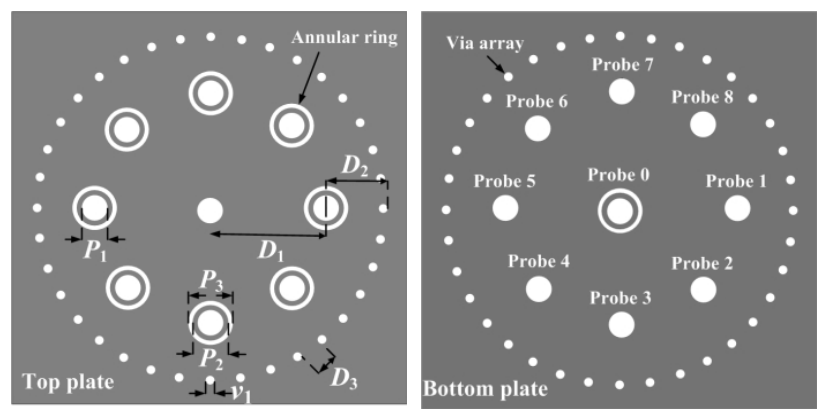

(a) SIW power divider

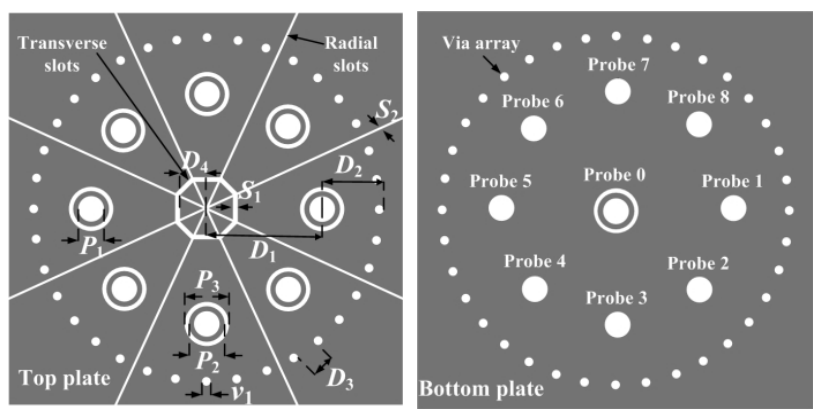

(b) Radial switch

Fig. 1. Configuration of the proposed structure.

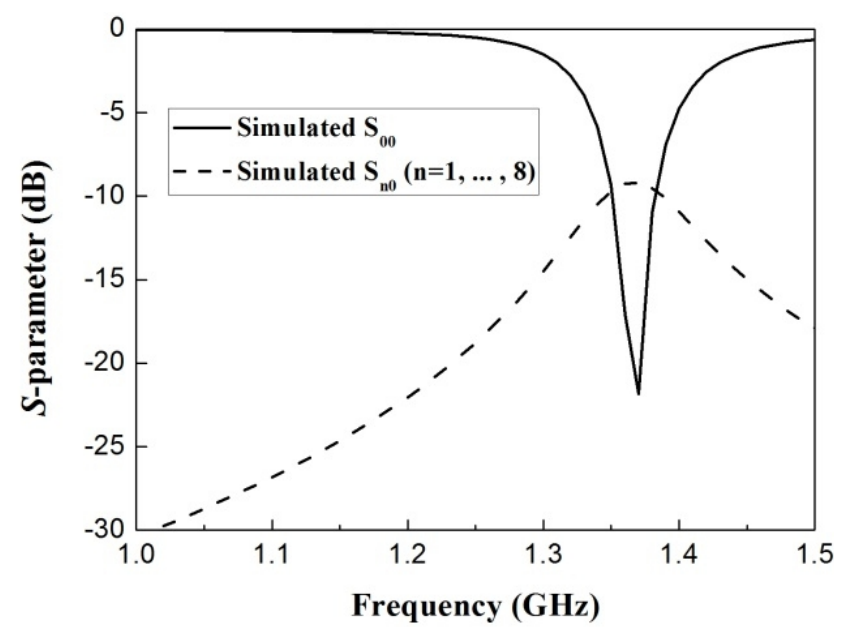

Fig. 2. Simulated $S$-parameter of SIW power divider. rings are placed at the junctions of coaxial probes to prohibit shorting between inner connectors of coaxial probes and the metal plate. As shown in Fig. 2, the designed structure divides input power equally with the center frequency of $1.37 \mathrm{GHz}$; the dimensions of this structure are summarized in Table 1. The radial switch requires that power be switched from the input to the target output probe and that the remaining seven probes be isolated. For this reason, radial slots onto the top plate of the designed power divider are introduced to separate the eight identical sectorial lines electrically. Therefore, each sectorial line is composed of an input probe and an output probe. The width of the radial slots is chosen to be as narrow as possible, in order to maintain a closed cavity structure. The radial slots do not degrade the performance of the power divider. Transverse slots are also inserted onto the top plate of the proposed structure, as shown in Fig. 1(b), to mount switch components. The other transverse slots, not illustrated in Fig. 1(b), are placed close to the central probe as DC blocking capacitors. For the switching operation, the varactor diode is mounted on the transverse slots in order to offer a different input impedance, looking from the central probe to each sectorial line. The effects of the mounted varactor will be analyzed in the following section.

\section{2-2 Tapered Line Resonator with Capacitor}

Each sectorial line comprising a central probe and an output probe can be viewed as a two-port network. However, analysis of this geometry is not straightforward, due to junction discontinuities. The array of via holes, which create electrical walls on the circumferential walls of the SIW cavity, can be considered as a shorting via of each sectorial line. Thus, a shorted tapered-line resonator concept is introduced in this paper for determining the resonant frequency and the variation of input impedance.

As shown in Fig. 3, the varactor diode is mounted on the transverse slot and is connected in series to the tapered-line. The resonant frequency of the switch is determined by obtaining the input impedance at the slot, using transmission line theory as:

$$
Z_{i n}=Z_{n}+1 / j \omega C_{v}
$$

where $C_{v}$ is a capacitance offered by varactor and $Z_{n}$ is the impedance at the beginning of the tapered-line looking toward the end of shorted tapered-line. The impedance of the tapered-line can be calculated by considering it as a number of cascaded transmission lines with different widths; this is defined by 


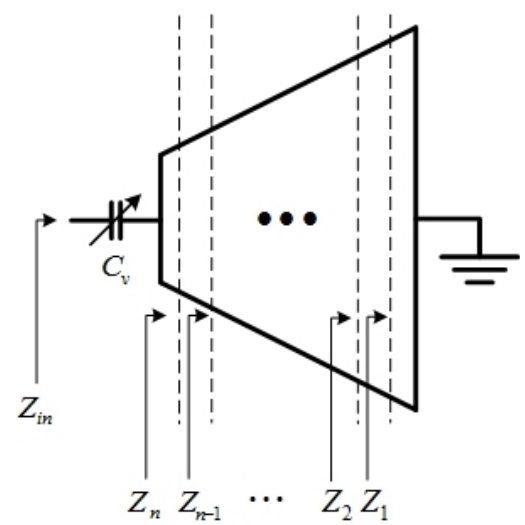

Fig. 3. Configuration of the shorted tapered line resonator.

$$
Z_{k}=Z_{0}^{k} \frac{Z_{L}^{k-1}+j Z_{0}^{k} \tan \left(\frac{\beta L}{n}\right)}{Z_{0}^{k}+j Z_{L}^{k-1} \tan \left(\frac{\beta L}{n}\right)} \quad(k=1, \ldots, n)
$$

where $n$ is the total number of segments, $L$ is the total length of tapered line, $Z_{0}^{k}$ is a characteristic impedance of $k$-th transmission line given by [14], and $Z_{L}^{k-1}$ is an input impedance of the previous section. The input impedance of the first section $(k=1)$, including shorting vias, can be written by

$$
Z_{1}=j Z_{0}^{1} \tan \left(\frac{\beta L}{n}\right)
$$

In Eq. (1), the resonance occurs when the imaginary part of the total input impedance vanishes. The calculated input impedances with a few varactor capacitances are plotted in Fig. 4. Inductance of the shorting via holes is ignored and the tapered-line is assumed to be lossless in the calculation.

As shown in Fig. 4, two resonant frequencies are

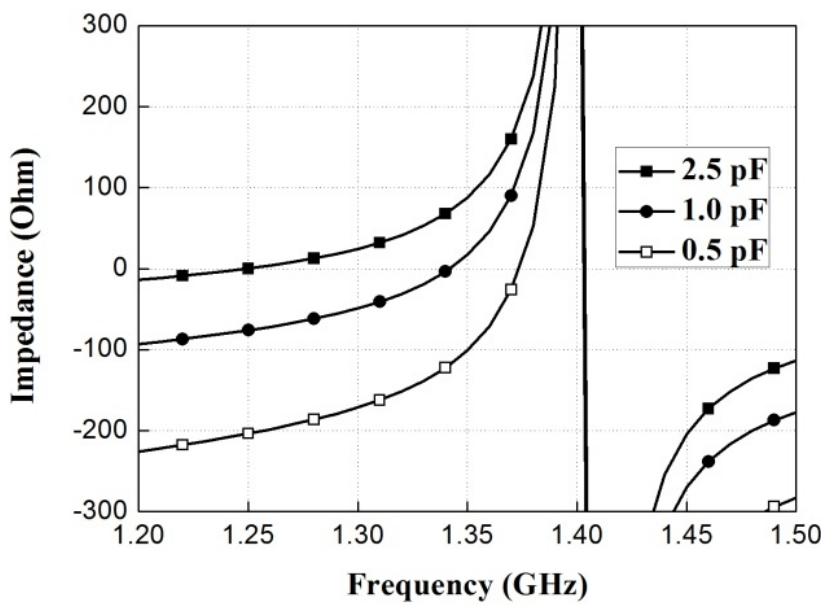

Fig. 4. Calculated imaginary part of input impedance.

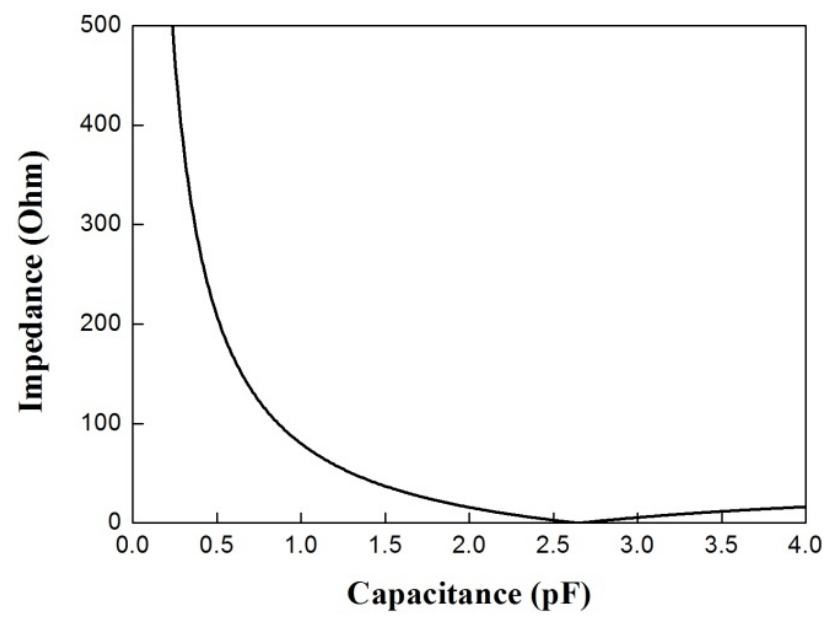

Fig. 5. Calculated magnitude of input impedance with varying capacitance at $1.25 \mathrm{GHz}$.

identified for each capacitance. The second resonant frequency, which corresponds to the operating frequency of the power divider, is the same irrespective of the varactor capacitances, because it depends on the dimension of the cavity resonator. Note that the resonant frequency is slightly higher than its operating frequency presented in Fig. 2; this is mainly due to the fact that the tapered-line from the central probe to transverse slots is not modeled. If the attached varactor provides $2.5 \mathrm{pF}$ of capacitance, the resonant frequency of a single tapered-line is around 1.25 GHz. For the switch operation, a target tapered-line is activated while the others are deactivated by the introduced capacitance on the transverse slots.

The input impedance looking at the front of the slot toward the shorted tapered-line is calculated and is plotted in Fig. 5 at a single frequency of $1.25 \mathrm{GHz}$, which is the resonant frequency of the tapered-line holding 2.5 $\mathrm{pF}$ of capacitance. Fig. 5 shows that the activated line is short-circuited when the line has $2.5 \mathrm{pF}$ of capacitance. However, the other lines with low capacitance can be seen as an open-circuit (high input impedance)

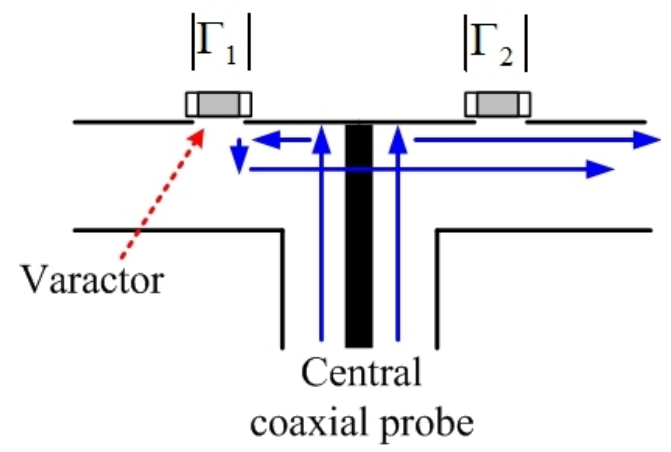

Fig. 6. Illustration of the power flow. 
and are deactivated at the switch operating frequency. The mechanism of the power flow is demonstrated in Fig. 6. The power fed by the central probe will propagate through the low input impedance path (low reflection coefficient). The power reflected at the slots due to the high input impedance (high reflection coefficient $\left.\left|\Gamma_{1}\right|\right)$ will be added to the low impedance path. The waves are also reflected by the remaining, but deactivated, lines and these waves may cancel out each other, because they are in phase and are travelling in the opposite direction, due to the symmetry of the proposed structure.

\section{Simulated and Measured Results}

This switch is fabricated with a substrate of $0.78 \mathrm{~mm}$ thickness, relative dielectric constant of 2.5 , and dielectric loss tangent of 0.001 . The parameters of the designed structure are summarized in Table 1, with the configuration shown in Fig. 1(b). The varactor diode used in this paper is M/A-Com MA46H071 (its capacitance $\left(C_{v}\right)$ swept from $0.45 \mathrm{pF}$ to $2.5 \mathrm{pF}$ with reverse bias). Because of the circumferential via array, the metallic plates containing output probes cannot be used for DC bias. Therefore, the metallic pads from the central probe to the slots are used for DC bias and a cathode of the varactor is connected with this pad. The $100 \mathrm{k} \Omega$ resistors are placed vertically on this pad for RF choke and directly connect to the bias wires.

Fig. 7 shows the measured $S$-parameter of the switch when zero bias is applied to all varactors. All insertion losses $\left(S_{n 0}, \mathrm{n}=1, \cdots, 8\right)$ are almost identical with the measured . The measured insertion loss is $9.75 \mathrm{~dB}$ including $9 \mathrm{~dB}$ power splitting. While the return loss is slightly more degraded than the return loss in Fig. 2, an equal power division is clearly observed. Zero reverse bias is applied to a single sectorial line, while $20 \mathrm{~V}$ of reverse bias is applied to deactivate the remaining seven lines. Under this condition, the measured insertion loss of the activated sectorial line is $3.9 \mathrm{~dB}$ at $1.23 \mathrm{GHz}$, as shown Fig. 8.

As discussed in the previous section, the tapered-line with $2.5 \mathrm{pF}$ of capacitance will be short-circuited and be activated around 1.25 GHz. The measured results are in good agreement with the calculated ones except for the loss characteristics. The loss includes the parasitic of the

Table 1. Dimension of eight-way radial switch (Unit: $\mathrm{mm}$ ).

\begin{tabular}{|c|c|c|c|c|c|}
\hline Parameter & $D_{1}$ & $D_{2}$ & $D_{3}$ & $D_{4}$ & $p_{1}$ \\
\hline Values & 39.85 & 14.6 & 13.6 & 2.8 & 0.8 \\
\hline Parameter & $p_{2}$ & $p_{3}$ & $v_{1}$ & $s_{1}$ & $s_{2}$ \\
\hline Values & 1.6 & 2.2 & 1.0 & 1.0 & 0.1 \\
\hline
\end{tabular}

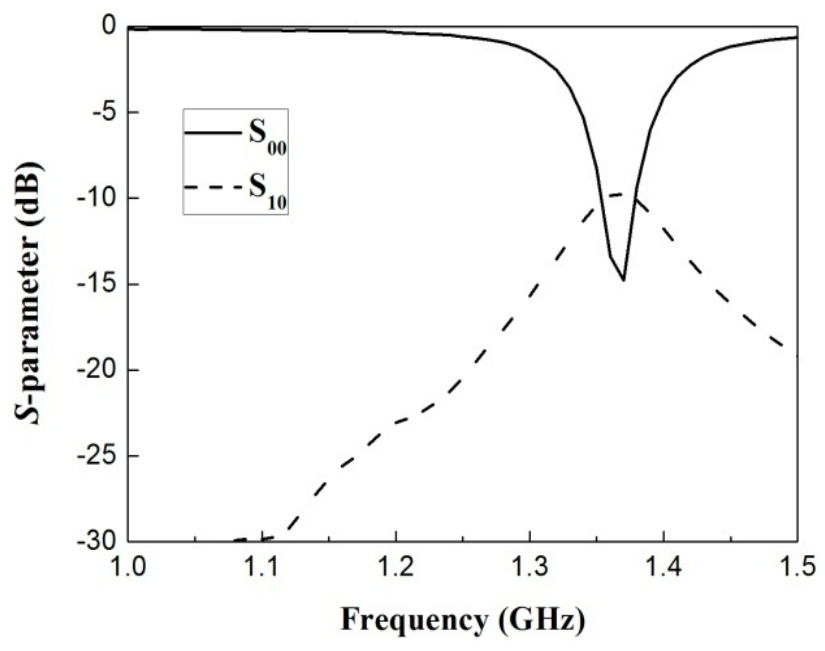

Fig. 7. Measured $S$-parameter of the proposed structure operating as power divider $\left(V_{R}=0 \mathrm{~V}\right)$.

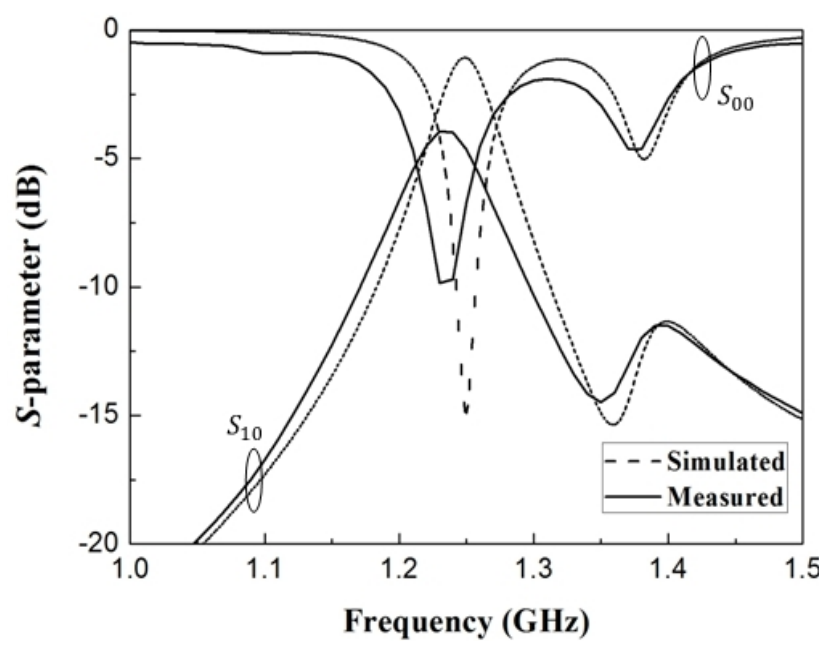

Fig. 8. Simulated and measured $S$-parameter (a single sectorial line is activated $\left(V_{R}=0 \mathrm{~V}\right)$, and other lines are isolated $\left(V_{R}=20 \mathrm{~V}\right)$.

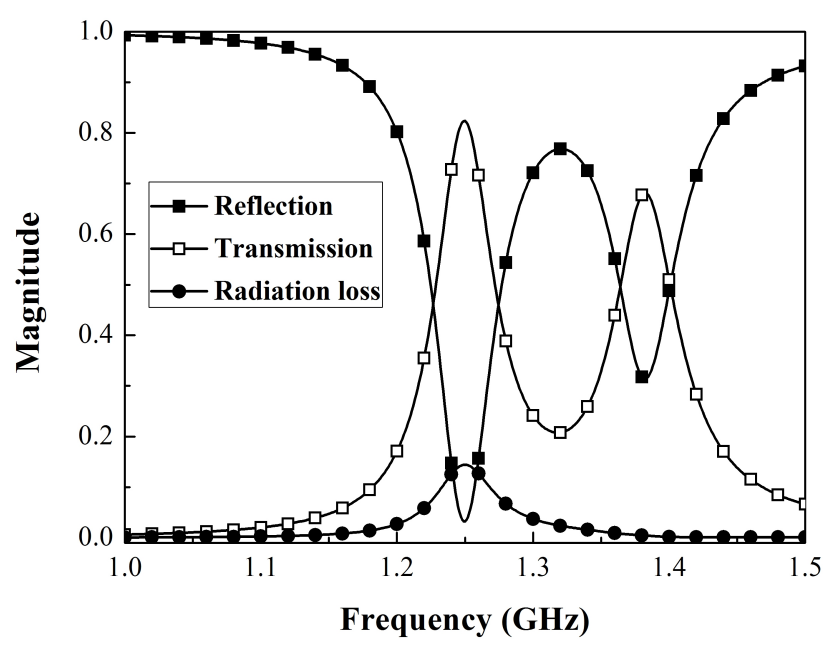

Fig. 9. Simulated loss mechanisms. 
varactor and the radiation loss. In the simulation, the varactor is modeled as an ideal capacitor. However, the series resistance $(=0.9 \Omega)$ of the varactor causes about $1 \mathrm{~dB}$ degradation. In addition, the proposed structure is simulated without dielectric and conductor losses to analyze radiation loss. If the network is lossless, energy conservation requires that the scattering matrix is unitary [14] and the results of the loss mechanisms are plotted in Fig. 9. Reflection, transmission, and radiation are defined as

$$
\begin{aligned}
& \text { Reflection }=\left|S_{00}\right|^{2} \\
& \text { Transmission }=\sum_{i=1}^{8}\left|S_{i 0}\right|^{2} \\
& \text { Radiation }=1-\left|S_{00}\right|^{2}-\sum_{i=1}^{8}\left|S_{i 0}\right|^{2} .
\end{aligned}
$$

As shown in Fig. 9, a radiation loss occurs due to the introduced slot lines. This radiation loss mainly causes the high insertion loss of the switch. The measured isolation of the remaining deactivated lines is higher than $20 \mathrm{~dB}$ at the operating bandwidth (shade region) of the switch, as shown in Fig. 10.

The performance of each line is measured while all remaining lines are deactivated, as shown in Fig. 11. The measured return and insertion losses of the worst case (port 8) are $7.86 \mathrm{~dB}$ and $5 \mathrm{~dB}$, respectively. The measured difference is due to fabrication errors and the performance variation of the varactor diode with bias states.

Nevertheless, the most of measured results show very good consistency. A photograph of the fabricated radial switch is shown in Fig. 12.

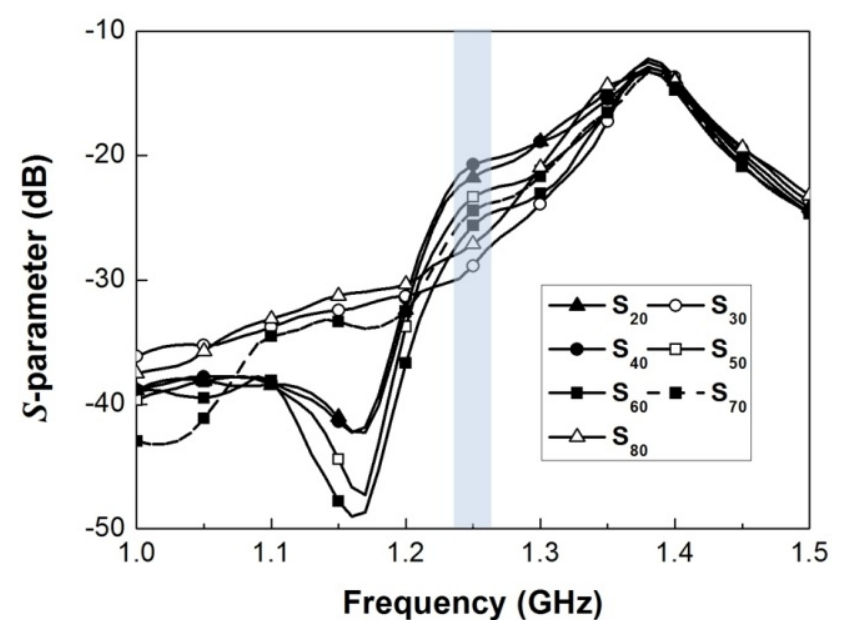

Fig. 10. Measured isolation $S$-parameter of deactivated lines.

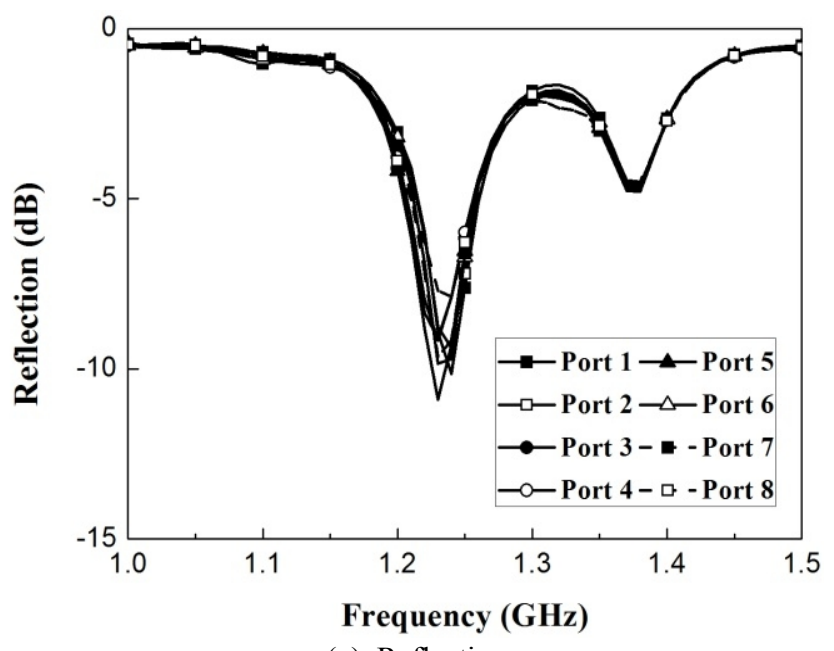

(a) Reflection

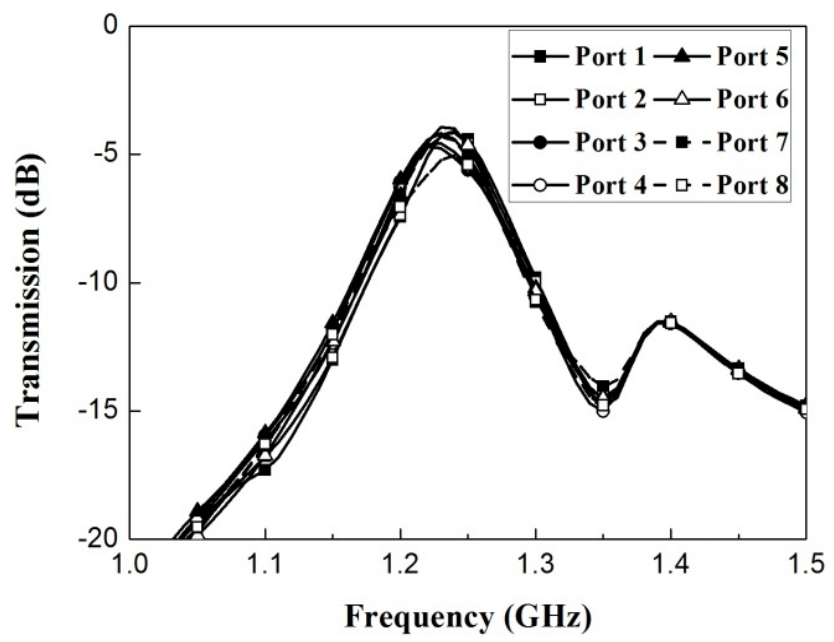

(b) Transmission

Fig. 11. Measured $S$-parameter of other lines named by port (1 to 8$)$.

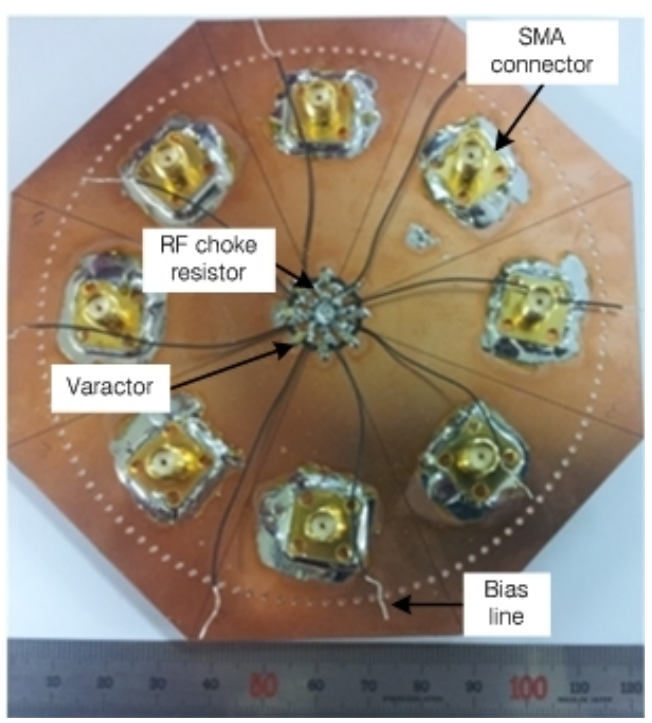

Fig. 12. Photograph of the proposed radial switch. 


\section{Conclusion}

A new eight-way radial switch based on a SIW radial cavity power divider is presented in this paper. The eight sectorial lines are separated by introducing radial slots, and these lines are able to control independently and electrically. The eight identical varactor diodes are mounted on transverse slots to provide different input impedances. Excellent performance of the power divider has been observed without reverse bias, revealing 0.75 $\mathrm{dB}$ of insertion loss at the center frequency. When the proposed structure operates as a switch circuit, the input power flows to the activated line that possesses low input impedance and is reflected by the deactivated lines holding high input impedance. The input impedance and the change in resonant frequency are calculated by assuming the sectorial lines as a tapered transmission line. The measured insertion loss is $3.9 \mathrm{~dB}$ and the isolation is higher than $20 \mathrm{~dB}$. The unwanted radiation loss arises from the introduced slots. Thus, extra care must be taken to reduce the radiation loss by careful design of the slot lines.

"This research was supported by the MKE (The Ministry of Knowledge Economy), Korea, under the ITRC (Information Technology Research Center) support program supervised by the NIPA (National IT Industry Promotion Agency)" (NIPA-2012-H0301-121001).

\section{References}

[1] J. A Ruiz-Cruz, M. M. Fahmi, and R. R. Mansour, "Generalized multiport waveguide switches based on multiple short-circuit loads in power-divider junctions," IEEE Transactions on Microwave Theory and Techniques, vol. 59, no. 12, pp. 3347-3355, Dec. 2011.

[2] A. J. Paffard, "O-band fast p-i-n-diode switch," Electronics Letters, vol. 12, no. 11, p. 272, May 1976.

[3] I. K. Joo, I. B. Yeom, and J. H. Park, "A PIN diode switch with high isolation and high switching speed," Journal of The Korean Institute of Electromagnetic Engineering and Science, vol. 16, no. 2, pp. 167-173, Feb. 2005.

[4] D. W. Kim, W. Kim, and B. B. Kim, "Implementation of high-power PIN diode switch modules and high-speed switch driver circuits for wibro base stations," Journal of The Korean Institute of Electromagnetic Engineering and Science, vol. 18, no. 4, pp. 364-371, Apr. 2007.

[5] R. S. Varnes, M. E. Blalkowski, "A switched radial divider for an L-band mobile satellite antenna," 25th European Microwave Conference, vol. 2, pp. 10371041, Sep. 1995.

[6] N. C. Karmakar, M. E. Bialkowski, "A beam-forming network for a circular switched-beam phased array antenna," IEEE Microwave Wireless Component. Letters, vol. 11, no. 1, pp. 7-9, Jan. 2001.

[7] Q. Wang, M. Lecours, and C. Vergnolle, "Criteria for wide-band radial switch design," IEEE Transactions on Microwave Theory and Techniques, vol. 49, no. 1, pp. 128-132, Jan. 2001.

[8] S. Pranonsatit, A. S. Holmes, I. D. Robertson, and S. Lucyszyn, "Single-pole eight-throw RF mems rotary switch," Journal of Microelectromechanical Systems, vol. 15, no. 6, pp. 1735-1744, Dec. 2006.

[9] M. Daneshmand, R. R Mansour, and N. Sarkar, "RF MEMS waveguide switch," IEEE Transactions on Microwave Theory and Techniques, vol. 52, no. 12, pp. 2651-2657, Dec. 2004.

[10] G. M. Rebeiz, J. B. Muldavin, "RF MEMS switches and switch circuits," IEEE Microwave Magazine, vol. 2, no. 4, pp. 59-71, Dec. 2001.

[11] K. Song, Y. Fan, and Y. Zhang, "Eight-way substrate integrated waveguide power divider with low insertion loss," IEEE Transactions on Microwave Theory and Techniques, vol. 56, no. 6, pp. 14731477, Jun. 2008.

[12] Y. P. Hong, Y. J. An, and J. G. Yook, "Differential radial power combiner using substrate integrated waveguide," Electronics Letters, vol. 46, no. 24, pp. 1607-1608, Nov. 2010.

[13] Y. P. Hong, J. G. Yook, "Varactor-tuned radial power divider with SIW technology," IEICE Transactions on Electronics, vol. E94-C, no. 12, pp. 19021905, Dec. 2011.

[14] D. M. Pozar, Microwave Engineering, 3rd Ed. Hoboken, NJ: J. Wiley, 2005. 


\section{Dong-Mook Lee}

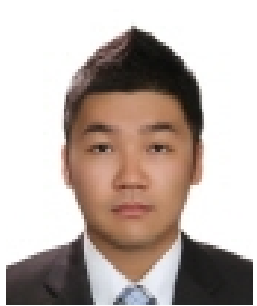

was born in Seoul, Korea, in 1984. He received the B.S. degree in physics from Yonsei University, Wonju, Korea, in 2010, and currently working toward the M.S degree in electrical engineering at Yonsei University. His research interests include RF passive components such as antenna, and power combiner/divider.

\section{Yong-Jun An}

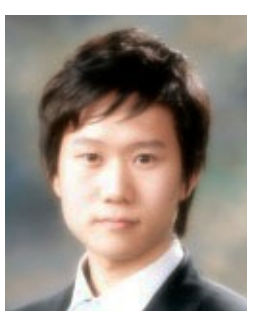

was born in Seoul, Korea, in 1986. He received the B.S. degree from Yonsei University, Seoul, Korea, in 2009, and currently working toward the Ph.D. degree in electrical engineering at Yonsei University. His research interests include RF system, antenna, and RF Integrate circuits.

\section{Jong-Gwan Yook}

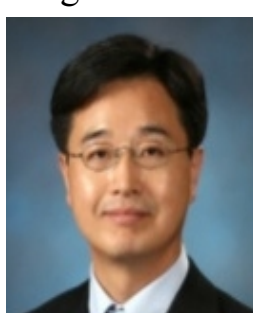

(S'89 M'97) was born in Seoul, Korea. He received the B.S. and M.S. degrees in electronics engineering from Yonsei University, Seoul, Korea, in 1987 and 1989, respectively, and the Ph.D. degree from The University of Michigan, Ann Arbor, MI, in 1996. He is currently a Professor with the School of Electrical and Electronic Engineering, Yonsei University. His main research interests are in the areas of theoretical/numerical electromagnetic modeling and characterization of microwave/millimeter-wave circuits and components, design of radio frequency integrated circuits (RFIC) and monolithic microwave integrated-circuit (MMIC), analysis and optimization of high-frequency highspeed interconnects, including RF microelectromechanical systems (MEMS), based on frequency as well as time-domain fullwave methods, and development of numerical techniques. Recently, his research team is developing various biosensors, such as carbon-nano-tube RF biosensor for nanometer size antigen- antibody detection as well as remote wireless vital signal monitoring sensors. 